\title{
The Central Role and Possible Mechanisms of Bacterial DNAs in Sepsis Development
}

\author{
Zhenxing Cheng $\mathbb{D}^{1,},{ }^{1,2}$ Simon T. Abrams, ${ }^{1}$ James Austin, ${ }^{1}$ Julien Toh, ${ }^{3}$ Susan Siyu Wang, \\ Zhi Wang, ${ }^{2}$ Qian Yu, ${ }^{2}$ Weiping Yu $\mathbb{D}^{2}$, Cheng Hock Toh $\mathbb{D}^{1,5}$ and Guozheng Wang ${ }^{1,2}$ \\ ${ }^{1}$ Department of Clinical Infection, Microbiology and Immunology, Institute of Infection and Global Health, The University \\ of Liverpool, Liverpool, L69 7BE, UK \\ ${ }^{2}$ Medical School, Southeast University, Nanjing 21009, China \\ ${ }^{3}$ Wirral University Teaching Hospitals NHS Foundation Trust, Arrowe Park Road, Upton, Wirral CH49 5PE, UK \\ ${ }^{4}$ Royal London Hospital, Whitechapel Rd, Whitechapel, London E1 1FR, UK \\ ${ }^{5}$ Liverpool University Hospitals NHS Foundation Trust, Liverpool L7 8XP, UK
}

Correspondence should be addressed to Weiping Yu; yuweiping@seu.edu.cn, Cheng Hock Toh; toh@liv.ac.uk, and Guozheng Wang; g.wang@liverpool.ac.uk

Received 2 May 2020; Accepted 20 July 2020; Published 31 August 2020

Academic Editor: Ronald Gladue

Copyright ( $) 2020$ Zhenxing Cheng et al. This is an open access article distributed under the Creative Commons Attribution License, which permits unrestricted use, distribution, and reproduction in any medium, provided the original work is properly cited.

\begin{abstract}
The pathological roles of bacterial DNA have been documented many decades ago. Bacterial DNAs are different from mammalian DNAs; the latter are heavily methylated. Mammalian cells have sensors such as TLR-9 to sense the DNAs with nonmethylated CpGs and distinguish them from host DNAs with methylated CpGs. Further investigation has identified many other types of DNA sensors distributed in a variety of cellular compartments. These sensors not only sense foreign DNAs, including bacterial and viral DNAs, but also sense damaged DNAs from the host cells. The major downstream signalling pathways includeTLR-9MyD88-IKKa-IRF-7/NF- $\kappa$ B pathways to increase IFN/proinflammatory cytokine production, STING-TBK1-IRF3 pathway to increase IFN-beta, and AIM2-ASC-caspas-1 pathway to release IL-1beta. The major outcome is to activate host immune response by inducing cytokine production. In this review, we focus on the roles and potential mechanisms of DNA sensors and downstream pathways in sepsis. Although bacterial DNAs play important roles in sepsis development, bacterial DNAs alone are unable to cause severe disease nor lead to death. Priming animals with bacterial DNAs facilitate other pathological factors, such as LPS and other virulent factors, to induce severe disease and lethality. We also discuss compartmental distribution of DNA sensors and pathological significance as well as the transport of extracellular DNAs into cells. Understanding the roles of DNA sensors and signal pathways will pave the way for novel therapeutic strategies in many diseases, particularly in sepsis.
\end{abstract}

\section{Introduction}

Damage-associated molecular patterns (DMAPs), including extracellular histones and DNAs, neutrophil extracellular traps (NETs) and other factors, mediate multiple organ injury and play key roles in critical illnesses, particularly in sepsis [13]. Extensive immune cell death often occurs in sepsis and becomes a major source of DMAPs $[4,5]$, but the underlying molecular mechanism is not clear. We found that bacterial DNAs play a major role in priming immune system and lead to extensive death of immune cells if a second hit appears.
However, this is a neglected field because many reports on DNA sensors and signalling pathways mainly focus on cancer and viral infection [6-8]. Here, we will review the available literatures of DNA sensors and signalling pathways that are related to bacterial infection and sepsis.

During bacterial infections, bacterial DNA is released after bacterial breakdown and enters the circulation. The DNA in circulation can then be rapidly cleared by the spleen and the liver with a half-life $\left(t^{1 / 2}\right)$ of approximately 4 minutes $[9,10]$. However, if the bacterial infection cannot be controlled, large quantities of bacterial DNA could continuously 
enter the circulation and trigger severe immune responses [11-13]. Circulating DNA taken up by the spleen stimulates lymphocytes to release cytokines [14]. High levels of circulating bacterial DNAs have a direct association with the metrics of clinical severity, suggesting that bacterial DNA levels are highly reflective of bacterial load [15]. Bacterial DNAtriggered immune responses play important roles in the elimination of human pathogens [16-18].

\section{Types of DNAs in Inflammation}

CpG motifs in bacterial DNAs trigger B cell activation [11]. In eukaryotic DNA, CpG motifs have a high degree of methylation, which may allow the host to distinguish them from the bacterial DNA [19], which is largely unmethylated. Methylation of CpG residues blocks their capacity of stimulating immune responses [20]. Bacterial DNA has been demonstrated to stimulate natural killer $(\mathrm{NK})$ cells to increase lytic activities and interferon $\gamma($ IFN $-\gamma)$ production, stimulate lymphocytes to secrete interleukin 6 and 12 (IL-6, IL-12) as well as IFN- $\gamma$, stimulate macrophages to produce tumour necrosis factor $\alpha$ (TNF- $\alpha)[11,12,21-24]$, and activate neutrophils [25]. Elevated TNF- $\alpha$ promotes the development of shock, and elevated IFN- $\gamma$ primes macrophage and natural killer (NK) cells to increase the toxicity of lipopolysaccharides (LPS) $[23,24,26-28]$. In the presence of DNA-binding proteins, such as HMGB1, which is a CpG-DNA-binding protein, B lymphocyte (B cells) activation is significantly enhanced either by bacterial DNA [29] or by a synthesized 6-base nucleotide motif of an unmethylated CpG dinucleotide flanked by 2 purines $\left(5^{\prime}\right)$ and 2 pyrimidines $\left(3^{\prime}\right)$. This nucleotide motif is present 20 times more in bacterial DNA than in vertebrate DNA $[11,30,31]$. The sequence specificity was demonstrated in direct DNA immunization using plasmid DNA carrying the necessary sequences for immune activation [32-34].

\section{Receptors and Signal Pathways}

Mammalian cells express a variety of DNA sensors as one of the first lines of defence against infection $[35,36]$. TLR-9 is a major receptor that recognises bacterial DNA and signals in host cells [37]. The TLR family are pattern recognition receptors activated by diverse conserved components of pathogens called pathogen-associated molecular patterns (PAMPs). In vertebrates, strong selective pressure maintains the highly conservative TLR receptors' recognition of and response to PAMPs [38]. TLR-9 receptors are expressed in many tissues including the spleen, where it is expressed most abundantly, and in many types of immune cells, including macrophages, lymphocytes, dendritic cells, natural killer cells, neutrophils, and other antigen-presenting cells [39]. UNC93B1 [40-42], PRAT4A [43, 44], and adaptor protein AP3 [45] transport TLR-9 from the endoplasmic reticulum to the endosome or the lysosome, where TLR-9 is matured after cleavage of its ectodomain by cathepsins [41, 46, 47]. Specific innate immune cells take up extracellular bacterial materials into endosomes and phagosomes, where the N-terminal domain of TLR- 9 senses the unmethylated CpG DNA motifs $[48,49]$. HMGB1 also forms complexes with TLR-9, and this causes translocation of TLR-9 to endosomes in response to CpG-DNA and CpG-oligodeoxynucleotides (CpG-ODN), which ultimately triggers cytokine responses [29]. CpGDNA activates TLR-9 to generate signalling through myeloid differentiation primary response gene 88 (MyD88), which in turn enhance IFN expression and activate proinflammatory and antimicrobial signals via mitogen-activated protein kinase $(\mathrm{MAPK})$ and/or nuclear factor $\kappa \mathrm{B}(\mathrm{NF}-\kappa \mathrm{B})$ pathways $[50,51]$. However, different cell types may vary in downstream pathways, for instance, in plasmacytoid DCs, myddosome forms to activate IRF-7 and increase IFN $\alpha$ production [52]. In contrast, macrophages rarely produce type-1 IFN in response to TLR9 ligands unless using DOTAP as the transfection reagent to deliver CpG-DNA into the cells [53]. However, TLR-9 and TLR-4 crosstalk amplifies macrophage responses through activation of the c-Jun $\mathrm{N}$-terminal kinases (JNKs) [54]. In lymphocytes, ligand-inducible high expression of TLR-9 was found in activated B lymphocytes [55]. DNA-containing antigen-triggered B cell responses rely on a TLR-9-dependent immune checkpoint [56].

Cyclic GMP-AMP synthase (cGAS), a cytosolic DNA sensor [57-59] also senses CpG DNA. However, cGAS recognises cytosolic double-stranded DNA in a sequence-independent manner and cannot distinguish microbial DNA from host DNA. cGAS-binding DNA produces cyclic GMP-AMP (cGAMP) to activate STING and signal via TBK1 and IFN regulatory factor (IRF)-3 to induce expression of type I IFN [60-62]. This signal also activates the NF- $\kappa \mathrm{B}$ pathway to enhance inflammatory cytokine release, including TNF- $\alpha$ and IL-6, which mediate inflammatory and antimicrobial responses [63-68]. STING also activates stress and cell death pathways that lead to $\mathrm{T}$ cell [69] and B cell death [70]. In chicken cells, DEAD box polypeptide 41 (DDX41) is an important DNA sensor to induce IFN production, which also depends on the STING pathway [71]. These observations strongly suggest that signalling through the STING pathway is a very important response to both cytosolic viral and bacterial DNA and mediates IFN-dependent innate immunity [72]. A STING-independent DNA sensing pathway (SIDSP) has also been identified. For example, DNA damage response protein DNA-PK was reported recently as the primary sensor in the SIDSP, which mainly activates the pathway via HSPA8/HSC70 in humans but is absent from mouse cells [73].

Absent in melanoma 2 (AIM2), a protein with a $\mathrm{N}$ terminal pyrin domain and a C-terminal HIN-200 domain, is an important component of the inflammasome [74, 75]. AIM2 senses cytoplasmic DNA to activate ASC (apoptosisassociated speck like protein) pyroprotosomes and caspase1 to ultimately cause cell death [76-81]. LL37 peptide can neutralize cytosolic DNA and block AIM2-mediated inflammasome activation [82]. IFI16 is another member of the HIN family and binds viral DNA to regulate IL- $1 \beta$ maturation, but does not associate with ASC [83]. IFI16 is predominantly a nuclear protein which translocates to the cytosol to recognize DNA and induces IFN- $\beta$ production. IFI16 also forms complexes with viral DNA in the nucleus, and these complexes translocate to the cytosol to trigger STINGdependent signalling [83-88]. Many other DNA sensors have been reported, including DNA-dependent activator of IFN- 


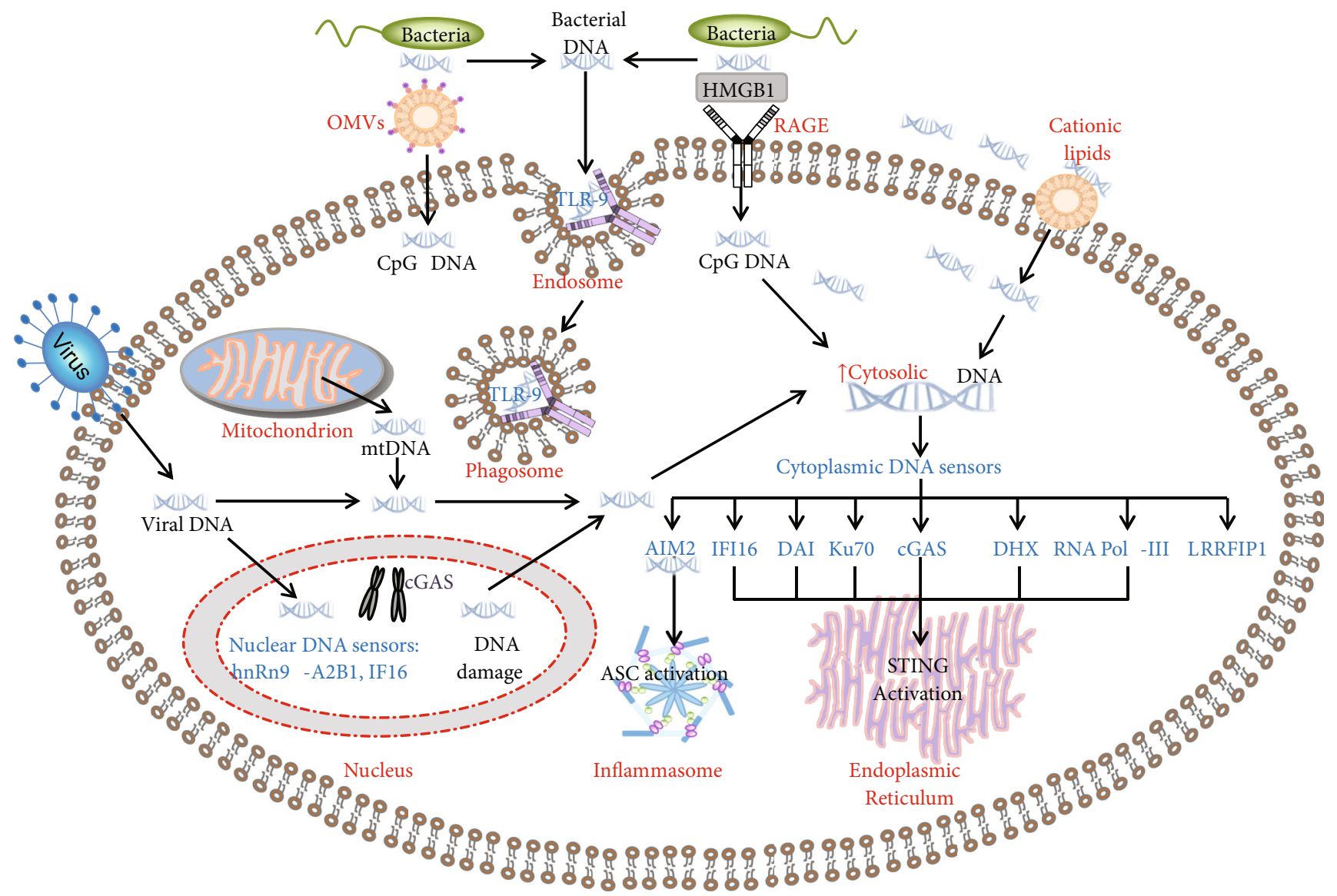

FIgURE 1: Compartmental distribution of DNA sensors and internalization of external DNAs. TLR-9 is the most important sensor for bacterial DNAs and is located on the membrane of endosomes and phagosomes. AIM2 binds to cytosol DNAs, which initiate the assembly of inflammasomes by activating ASC. Many other cytosolic DNA sensors have been identified, and most of them activate STING pathways, including cGAS, IFI16, DAI, Ku70, DHX9, DHX36, DHX41, and RNA Pol-III. LRRFIP1 is also a cytosolic DNA sensor but it mainly triggers the beta-catenin pathway. cGAS and IFI16 also exist in cell nuclei but cGAS binds to chromosome to enhance its stability whist nuclear cGAS and IFI16 plus hnRNP-A2B1-DNA can detect viral DNAs which enter nuclei and form complexes with DNAs. The complexes are exported to the cytosol to activate STING pathways. Most DNA sensors are situated inside cells, and the extracellular DNAs from virus, bacteria, or host cells enter cells by many different ways, including endocytosis and phagocytosis, or are facilitated by HMGB1-RAGE, cationic lipids, or OMVs. Viruses enter cells via different membrane proteins.

regulatory factor (DAI, also called Z-DNA binding protein1, ZBP1) [89], leucine-rich repeat interacting protein-1 (Lrrfip1) [90], RNA polymerase III [91, 92], Ku70, and DExD/H box helicases (DHX9 and DHX36) [93]. DAI recognizes both the B-form and Z-form of cytosolic ds-DNA. LRRFIP1 induces IFN production via a $\beta$-catenin-dependent pathway [90], whilst RNA polymerase III transcribes DNA into $5^{\prime}$-ppp RNA to activate RIG-1-STING pathway, which triggers IFN production [91, 92]. Ku70 acts as a cytosolic DNA sensor to promote type III IFN- $\lambda 1$ production via activation of IRFland IRF7 [94]. DHX36 detects CpG-A DNA using the DEAH domain to mediate IFN- $\alpha$ production via the MyD88-IRF7 pathway, whilst DHX9 senses CpG-B DNA using the DUF domain to increase TNF- $\alpha$ and IL-6 production via the MyD88-NF- $\kappa \mathrm{B}$ pathway $[93,95,96]$. Recently, heterogeneous nuclear ribonucleoprotein A2/B1 (hnRNP-A2B1) has been shown to recognise viral doublestranded DNA inside the cell nucleus but not the host DNA that is packed as nucleosomes. The hnRNP-A2B1-
DNA complexes translocated into the cytosol induce IFN production via STING-dependent pathways [97]. The major pathways initiated by DNA sensors are summarized in Figure 1.

\section{Compartmental Distribution of DNA Sensors}

As mentioned above, each DNA sensor is located in a specific compartment (see Figure 1). TLR-9 exists in endosomes and phagosomes to detect DNAs that entered the cell via endocytosis or phagocytosis [53]. There are many cytosolic DNA sensors, including DAI, Ku70, RNA pol III, DHX9/36, and LRRFIP1, to detect cytosolic DNA. AIM2 is located in the inflammasome, hnRNP-A2B1 in nucleus, and IFI16 in the nuclear plasma. cGAS attached to the plasma membrane by N-terminal phosphoinositide-binding domain detects viral DNAs, whilst mutant cGAS in the cytosol mainly responds to host stress instead of viral DNAs [98]. In contrast, chromatin-bound cGAS inhibits DNA repair to increase 


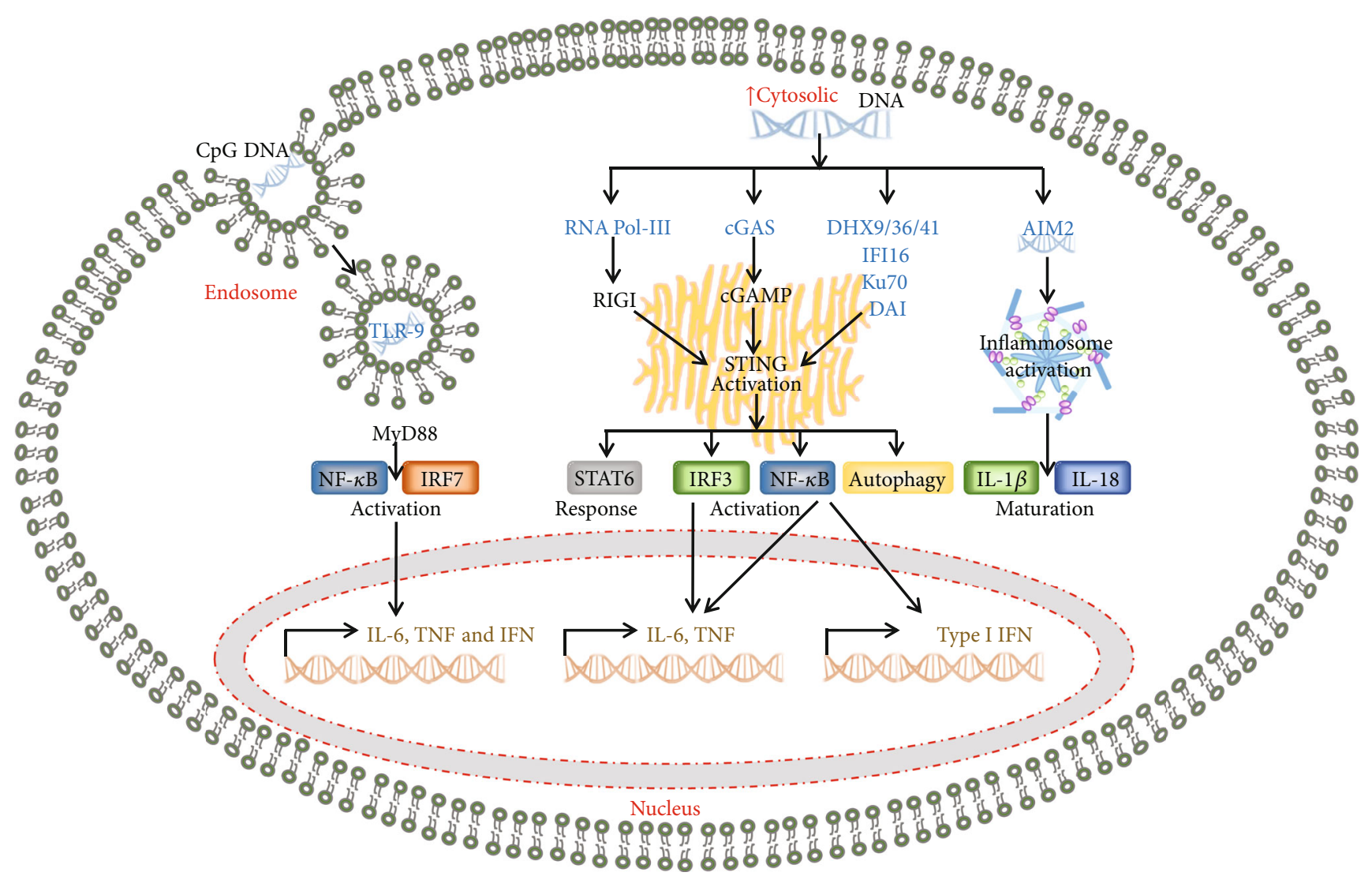

FIGURE 2: Major signalling pathways of DNA sensors. TLR-9-MyD88, STING, and AIM2-ASC are the major pathways initiated by DNA sensors. TLR9 preferentially binds bacterial and viral DNAs to trigger NF- $\kappa$ B and IRF7 signaling cascades via MyD88 adaptor and lead to a proinflammatory cytokine response. STING is a common pathway of many DNA sensors to induce IFN and other proinflammatory cytokines. AIM2-ASC induces inflammasome assembly and activation to process IL-1beta and IL-18.

genome stability and promotes cell death [99], indicating the importance of compartmentalisation of DNA sensor proteins.

DNA sensors distributed in a variety of cell compartments may play an important role in sensing bacterial and viral DNA that enter cells via different mechanisms so as to ensure swift recognition by the host. However, T and B cells, unlike macrophages, do not take up DNA which limits their response to bacterial DNAs, particularly high molecular weight DNA [100]. When DNA is physically linked to hen egg lysozyme (HEL), the resulting complex was efficiently taken up by B cells in the experimental setting [100]. In bacterial infections, besides endocytosis, many other factors, such as HMGB1-RAGE [101], cationic lipids [102], and outer membrane vesicles (OMVs) [103], may facilitate bacterial DNA uptake by host cells and trigger DNA sensors and downstream signal pathways.

\section{STING Plays a Central Role in Both Exogenous and Endogenous DNA Sensing}

DNA-sensing receptor cyclic GMP-AMP synthase (cGAS) activating the STING pathway plays a central role in sensing and responding to cytosolic DNA from both exogenous and endogenous sources. Endogenous nuclear DNA damage caused by ionizing radiation, oxidative stress, drugs, telomere shortening, chromosome mis-segregation, mitochondrial damage, and viral/bacterial infection all lead to accumulation of DNA in the cytosol [104]. Once the DNA is exposed to the cGAS sensor, cGAS forms dimers and synthesizes cyclic guanosine monophosphate-adenosine monophosphate (cGAMP). The cGAMP acts as a second messenger to activate STING on the ER surface, which ultimately activates IRF3 and NF- $\kappa$ B through the kinases TBK1 and IKK, respectively, so as to increase expression of the cytokines including IFN and TNF- $\alpha$ [73, 104, 105] (see Figure 2). On the other hand, STING can also mediate IFI16 degradation by reducing type I interferon production [106]. Many other factors, such as cyclic di-nucleotides from intracellular pathogens, DHX41, RNA Pol-III, DAI, or IFI16, also activate STING pathway (Figure 1) and initiate different responses mediated by IRF3, NF- $\kappa$ B, STAT6 [107], and autophagy [108] pathways (Figure 2). Therefore, the cGASSTING pathway is an important regulator of free DNA resulting from infection, inflammation, or cancer [104, 109, 110].

Activation of the STING pathway not only causes cytokine release, but also induces apoptosis in immune cells, including in T and B lymphocytes [111], myeloid lineage cells, and in nonimmune cells, such as hepatocytes $[112,113]$ and cardiomyocytes [114], and in cancer cells $[70,110,115]$. STING pathway-induced apoptosis involves ER stress and Bak/Bax-mediated macropores in the 
mitochondrial outer membrane or leakage of lysosomal content into the cytosol $[69,114,116]$. Recent reports demonstrate that STING signalling plays an important role in the induction of necrosis via the synergistic effect between IFN and TNF- $\alpha$ pathways [118; 119].

\section{Syngeneic Effects with Other Toxic Factors}

Although bacterial CpG DNA triggers an immune response via DNA sensors, incubation of cells with the bacterial DNA or injection of DNA into mice is not sufficient to cause cell or animal death [117]. CpG DNA motifs were reported as sensitizing agents for lipopolysaccharide-induced toxic shock in animal models [117]. CpG-DNA and LPS can act synergistically to induce inflammatory cytokines and nitric oxide release, and to increase cell-surface DNA binding and internalisation in monocytes and macrophages [118-122]. CPG DNA and LPS synergistically induce TNF- $\alpha$ production via activation of NF- $\kappa \mathrm{B}[120,123]$. High doses of Dgalactosamine is lethal in animal models [124], whilst lower doses (nonlethal) are used to sensitize animals [125]. In sensitized mice, CpG DNA or LPS is able to induce acute liver injury via the mitochondrial apoptotic pathway involving TLR pattern recognition receptors [126-128]. Galactosamineinduced sensitization also contributes to endotoxin-induced immune response and lethality $[129,130]$.

\section{Roles of Bacterial DNAs in Disease}

Bacterial infection causes variety of diseases with different mechanisms and manifestations. The majority of bacteria do not enter host cells, but releases DNAs which are transported into cells via endocytosis or phagocytosis to meet TLR-9 receptor [37]. Some bacteria could enter host cells and release DNAs directly into cytosol to trigger cytosolic DNA sensors [131].

It was reported that bacterial DNA could cause septic shock via induction of high levels of TNF- $\alpha$ [132]. However, no confirmatory report has since been published. In general, bacterial DNA causes a variety of immune responses, but no lethal effects, although blocking TLR-9 reduces bacterial load, inflammation, and mortality in mouse poly-microbial sepsis [133]. Kukoamine B, a novel dual inhibitor of LPS and CpG-DNA, has been reported to be effective in treating animals with sepsis [134]. Intratracheal admission of synthetic CpG-ODNs leads to acute lung inflammation and injury with systemic inflammatory response via activation of TLR-9 [135, 136]. Bacterial DNA induces pulmonary damage via TLR-9, and suppressing CpG-ODNs and TLR-9 could significantly reduce inflammation in the mouse lungs $[137,138]$. The same effect has also been reported in cardiac injury and malfunction caused by bacterial DNA [10].

On the other hand, synthetic oligonucleotides have also been used as modulators of inflammation and immune response [139]. Single-stranded DNA containing CpG motifs have also been shown to induce innate immune responses, including production of poly-reactive immunoglobulin and the production of T helper 1 (TH1)-type as well as release of proinflammatory cytokines and chemokines [140] and to activate B lymphocytes [11]. The innate immune responses are able to increase host resistance to a wide range of pathogenic bacteria, viruses, and parasites, and therefore, $\mathrm{CpG}-$ ODNs have been tried with vaccines to enhance immunity [141]. When mice are exposed to bacterial DNA before hemorrhagic shock, the systemic inflammation and gut barrier loss via an IFN- $\gamma$-dependent route are strongly aggravated [142]. CpG ODNs has also been shown to stimulate protective innate immunity, enhance the complement system, protect immune cells, increase secretion of antibacterial antibody, and enhance phagocytosis against intracerebral E. coli K1 infection, pulmonary Klebsiella, or Staphylococcus aureus infections [143-148]. These observations indicate that there are both harmful and beneficial effects of bacterial DNA.

\section{Potential Diagnostic and Therapeutic Strategy and Reagents}

DNase, particularly DNase 1, has been used directly in many animal models to digest extracellular DNAs, including DNAs in dead bacteria and neutrophil extracellular traps (NETs) [149-151]. It is very likely that DNase 1 will be used clinically in the near future [152]. The other strategy is to target the sensors of bacterial DNAs and downstream pathways, mainly the TLR-9 and cGAS-STING pathways. As mentioned above, targeting TLR-9 improves the outcomes of mouse sepsis [133]. Targeting the TLR-9-MyD88 pathway is also used to regulate adaptive immune responses [153], but the majority of studies were focused on cancer [154]. Many drugs targeting TLR-9 and other TLRs are under clinical trials, mainly for cancer therapy $[153,155]$. However, targeting TLRs has demonstrated a great potential in sepsis management and infection control, as well as optimisation of vaccine efficacy $[156,157]$. STING-knockout mice developed less severe acute pancreatitis than wild type mice, whilst STING agonists cause more severe acute pancreatitis [158]. However, the STING ligand, c-di-GMP, can improve vaccination results for metastatic breast cancer [159]. Recently, the STING pathway has become much more attractive in targeting both inflammation and cancer [160]. STING agonists also reduce the burden of pancreatic cancer in mouse models [161]. As such, many STING pathway agonists and antagonists have been developed [162-164]. Although the science behind this pathway is becoming clearer, successful development of medication targeting this pathway appears a long way off.

Since the major outcome of DNA priming is the production of IFN- $\gamma$ and TNF- $\alpha$, which are the key cytokines of the generalized Shwartzman reaction [27], reports have been accumulated that demonstrate the roles of IFN- $\gamma$ and its receptors in animal models of sepsis and endotoxemia. IFN- $\gamma$-sensitized mice are more susceptible to LPS-induced mortality $[23,27,165]$. Thus, IFN- $\gamma-/$ - mice showed a significant reduction in infection-induced splenic cell apoptosis [166]. Moreover, IFN- $\gamma$ receptor-deficient mice gain resistance to LPS-induced septic shock [167]. Anti-IFN- $\gamma$ has also been demonstrated to have protective effects in animal models $[168,169]$. However, no successful clinical trials on sepsis have been reported yet, although some clinical trials 
on the roles of anti-IFN- $\gamma$ in sepsis-induced immune dysfunction are nearing completion. There is no evidence to demonstrate whether blocking the pathway of IFN- $\gamma$ production, such as using DNase 1, would be a better choice.

\section{Conclusion}

The roles of different DNAs and sensors are still a major area of scientific research. How these sensors and pathways are coordinated to function in different types of cells still needs further clarification. Moreover, the cross-talk between the inflammatory pathways and the cell proliferation or death pathways has been shown to be very important in elucidating the pathological mechanisms in the development of diseases. In sepsis, there is a big gap between bacterial DNA priming and immune cell death and subsequently disease development, although it is known that the priming process is very important. Thus, more work needs to be done both in vitro and in vivo to elucidate the signalling pathways and to identify the targets. Sepsis is a common disease with an unacceptable mortality rate, but no specific therapy is yet available. Clarification of the roles and molecular mechanisms of bacterial DNAs in development of sepsis may lead to the creation of new therapies to increase the survival of patients with sepsis.

\section{Conflicts of Interest}

The authors declare that the research was conducted in the absence of any commercial or financial interest.

\section{Acknowledgments}

This work was supported by the British Heart Foundation (PG/14/19/30751 and PG/16/65/32313) and Scholarships from China Scholarship Council (CSC: 20160609156) and the Southeast University (YBJJ1740) to Zhenxing Cheng.

\section{References}

[1] N. L. Denning, M. Aziz, S. D. Gurien, and P. Wang, "DAMPs and NETs in sepsis," Frontiers in Immunology, vol. 10, 2019.

[2] M. C. Gruda, K. G. Ruggeberg, P. O'Sullivan et al., "Broad adsorption of sepsis-related PAMP and DAMP molecules, mycotoxins, and cytokines from whole blood using CytoSorb ${ }^{\circledR}$ sorbent porous polymer beads," PLoS One, vol. 13, no. 1, article e0191676, 2018.

[3] J. Eppensteiner, J. Kwun, U. Scheuermann et al., "Damageand pathogen-associated molecular patterns play differential roles in late mortality after critical illness," JCI Insight, vol. 4, no. 16, article e127925, 2019.

[4] R. S. Hotchkiss, S. B. Osmon, K. C. Chang, T. H. Wagner, C. M. Coopersmith, and I. E. Karl, "Accelerated lymphocyte death in sepsis occurs by both the death receptor and mitochondrial pathways," The Journal of Immunology, vol. 174, no. 8, pp. 5110-5118, 2005.

[5] F. Pinheiro da Silva and V. Nizet, "Cell death during sepsis: integration of disintegration in the inflammatory response to overwhelming infection," Apoptosis, vol. 14, no. 4, pp. 509-521, 2009.
[6] J. Kwon and S. F. Bakhoum, "The cytosolic DNA-sensing cGAS-STING pathway in cancer," Cancer Discovery, vol. 10, no. 1, pp. 26-39, 2020.

[7] T. Abe, Y. Marutani, and I. Shoji, "Cytosolic DNA-sensing immune response and viral infection," Microbiology and Immunology, vol. 63, no. 2, pp. 51-64, 2019.

[8] S. R. Paludan and A. G. Bowie, "Immune sensing of DNA," Immunity, vol. 38, no. 5, pp. 870-880, 2013.

[9] P. Rumore, B. Muralidhar, M. Lin, C. Lai, and C. R. Steinman, "Haemodialysis as a model for studying endogenous plasma DNA: oligonucleosome-like structure and clearance," Clinical and Experimental Immunology, vol. 90, no. 1, pp. 56-62, 1992.

[10] P. Knuefermann, M. Schwederski, M. Velten et al., "Bacterial DNA induces myocardial inflammation and reduces cardiomyocyte contractility: role of toll-like receptor 9," Cardiovascular Research, vol. 78, no. 1, pp. 26-35, 2008.

[11] A. M. Krieg, A. K. Yi, S. Matson et al., "CpG motifs in bacterial DNA trigger direct B-cell activation," Nature, vol. 374, no. 6522, pp. 546-549, 1995.

[12] A. K. Yi, D. M. Klinman, T. L. Martin, S. Matson, and A. M. Krieg, "Rapid immune activation by CpG motifs in bacterial DNA. Systemic induction of IL-6 transcription through an antioxidant-sensitive pathway," Journal of Immunology, vol. 157, no. 12, pp. 5394-5402, 1996.

[13] G. S. Gilkeson, A. M. Pippen, and D. S. Pisetsky, "Induction of cross-reactive anti-dsDNA antibodies in preautoimmune NZB/NZW mice by immunization with bacterial DNA," The Journal of Clinical Investigation, vol. 95, no. 3, pp. 1398-1402, 1995.

[14] T. M. Chused, A. D. Steinberg, and N. Talal, "The clearance and localization of nucleic acids by New Zealand and normal mice," Clinical and Experimental Immunology, vol. 12, no. 4, pp. 465-476, 1972.

[15] S. J. Hackett, M. Guiver, J. Marsh et al., "Meningococcal bacterial DNA load at presentation correlates with disease severity," Archives of Disease in Childhood, vol. 86, no. 1, pp. 44-46, 2002.

[16] W. E. Paul, R. A. Seder, and M. Plaut, "Lymphokine and Cytokine Production by FceRI ${ }^{+}$Cells," Advances in Immunology, vol. 53, pp. 1-29, 1993.

[17] E. Bohn, J. Heesemann, S. Ehlers, and I. B. Autenrieth, "Early gamma interferon mRNA expression is associated with resistance of mice against Yersinia enterocolitica," Infection and Immunity, vol. 62, no. 7, pp. 3027-3032, 1994.

[18] F. P. Heinzel, M. D. Sadick, S. S. Mutha, and R. M. Locksley, "Production of interferon gamma, interleukin 2, interleukin 4 , and interleukin 10 by CD4+ lymphocytes in vivo during healing and progressive murine leishmaniasis," Proceedings of the National Academy of Sciences of the United States of America, vol. 88, no. 16, pp. 7011-7015, 1991.

[19] A. Bird, "The essentials of DNA methylation," Cell, vol. 70, no. 1, pp. 5-8, 1992.

[20] K. J. Stacey, M. J. Sweet, and D. A. Hume, "Macrophages ingest and are activated by bacterial DNA," Journal of Immunology, vol. 157, no. 5, pp. 2116-2122, 1996.

[21] S. Yamamoto, T. Yamamoto, S. Shimada et al., "DNA from bacteria, but not from vertebrates, induces interferons, activates natural killer cells and inhibits tumor growth," Microbiology and Immunology, vol. 36, no. 9, pp. 983-997, 1992. 
[22] D. M. Klinman, A. K. Yi, S. L. Beaucage, J. Conover, and A. M. Krieg, "CpG motifs present in bacteria DNA rapidly induce lymphocytes to secrete interleukin 6, interleukin 12, and interferon gamma," Proceedings of the National Academy of Sciences of the United States of America, vol. 93, no. 7, pp. 2879-2883, 1996.

[23] J. S. Cowdery, J. H. Chace, A. K. Yi, and A. M. Krieg, "Bacterial DNA induces NK cells to produce IFN-gamma in vivo and increases the toxicity of lipopolysaccharides," Journal of Immunology, vol. 156, no. 12, pp. 4570-4575, 1996.

[24] T. Sparwasser, T. Miethke, G. Lipford et al., "Macrophages sense pathogens via DNA motifs: induction of tumor necrosis factor-alpha-mediated shock," European Journal of Immunology, vol. 27, no. 7, pp. 1671-1679, 1997.

[25] A. . S. Trevani, A. Chorny, G. Salamone et al., "Bacterial DNA activates human neutrophils by a CpG-independent pathway," European Journal of Immunology, vol. 33, no. 11, pp. 3164-3174, 2003.

[26] M. J. Sweet, K. J. Stacey, D. K. Kakuda, D. Markovich, and D. A. Hume, "IFN-gamma primes macrophage responses to bacterial DNA," Journal of Interferon \& Cytokine Research, vol. 18, no. 4, pp. 263-271, 1998.

[27] L. Ozmen, M. Pericin, J. Hakimi et al., "Interleukin 12, interferon gamma, and tumor necrosis factor alpha are the key cytokines of the generalized Shwartzman reaction," The Journal of Experimental Medicine, vol. 180, no. 3, pp. 907-915, 1994.

[28] M. D. Halpern, R. J. Kurlander, and D. S. Pisetsky, "Bacterial DNA induces murine interferon-gamma production by stimulation of interleukin-12 and tumor necrosis factor-alpha," Cellular Immunology, vol. 167, no. 1, pp. 72-78, 1996.

[29] S. Ivanov, A. M. Dragoi, X. Wang et al., "A novel role for HMGB1 in TLR9-mediated inflammatory responses to CpG-DNA,” Blood, vol. 110, no. 6, pp. 1970-1981, 2007.

[30] L. R. Cardon, C. Burge, D. A. Clayton, and S. Karlin, "Pervasive CpG suppression in animal mitochondrial genomes," Proceedings of the National Academy of Sciences of the United States of America, vol. 91, no. 9, pp. 3799-3803, 1994.

[31] Y. Gruenbaum, H. Cedar, and A. Razin, "Restriction enzyme digestion of hemimethylated DNA," Nucleic Acids Research, vol. 9, no. 11, pp. 2509-2515, 1981.

[32] Y. Sato, M. Roman, H. Tighe et al., "Immunostimulatory DNA sequences necessary for effective intradermal gene immunization," Science, vol. 273, no. 5273, pp. 352-354, 1996.

[33] E. Raz, H. Tighe, Y. Sato et al., "Preferential induction of a Th1 immune response and inhibition of specific IgE antibody formation by plasmid DNA immunization," Proceedings of the National Academy of Sciences of the United States of America, vol. 93, no. 10, pp. 5141-5145, 1996.

[34] D. M. Klinman, G. Yamshchikov, and Y. Ishigatsubo, "Contribution of CpG motifs to the immunogenicity of DNA vaccines," Journal of Immunology, vol. 158, no. 8, pp. 3635-3639, 1997.

[35] N. Semenova, M. Bosnjak, B. Markelc, K. Znidar, M. Cemazar, and L. Heller, "Multiple cytosolic DNA sensors bind plasmid DNA after transfection," Nucleic Acids Research, vol. 47, no. 19, pp. 10235-10246, 2019.

[36] S. Akira, S. Uematsu, and O. Takeuchi, "Pathogen recognition and innate immunity," Cell, vol. 124, no. 4, pp. 783801, 2006.
[37] H. Hemmi, O. Takeuchi, T. Kawai et al., "A Toll-like receptor recognizes bacterial DNA," Nature, vol. 408, no. 6813, pp. 740-745, 2000.

[38] J. C. Roach, G. Glusman, L. Rowen et al., "The evolution of vertebrate Toll-like receptors," Proceedings of the National Academy of Sciences of the United States of America, vol. 102, no. 27, pp. 9577-9582, 2005.

[39] T. Kawasaki and T. Kawai, "Toll-like receptor signaling pathways," Frontiers in Immunology, vol. 5, 2014.

[40] Y. M. Kim, M. M. Brinkmann, M. E. Paquet, and H. L. Ploegh, "UNC93B1 delivers nucleotide-sensing toll-like receptors to endolysosomes," Nature, vol. 452, no. 7184, pp. 234-238, 2008.

[41] R. Fukui, S. Saitoh, F. Matsumoto et al., "Unc93B1 biases Toll-like receptor responses to nucleic acid in dendritic cells toward DNA- but against RNA-sensing," The Journal of Experimental Medicine, vol. 206, no. 6, pp. 1339-1350, 2009.

[42] B. L. Lee, J. E. Moon, J. H. Shu et al., "UNC93B1 mediates differential trafficking of endosomal TLRs," eLife, vol. 2, article e00291, 2013.

[43] K. Takahashi, T. Shibata, S. Akashi-Takamura et al., "A protein associated with Toll-like receptor (TLR) 4 (PRAT4A) is required for TLR-dependent immune responses," The Journal of Experimental Medicine, vol. 204, no. 12, pp. 29632976, 2007.

[44] T. Kiyokawa, S. Akashi-Takamura, T. Shibata et al., "A single base mutation in the PRAT4A gene reveals differential interaction of PRAT4A with Toll-like receptors," International Immunology, vol. 20, no. 11, pp. 1407-1415, 2008.

[45] E. Tomasello, K. Naciri, R. Chelbi et al., "Molecular dissection of plasmacytoid dendritic cell activation in vivo during a viral infection," EMBO Journal, vol. 37, no. 19, 2018.

[46] B. Park, M. M. Brinkmann, E. Spooner, C. C. Lee, Y. M. Kim, and H. L. Ploegh, "Proteolytic cleavage in an endolysosomal compartment is required for activation of Toll-like receptor 9," Nature Immunology, vol. 9, no. 12, pp. 1407-1414, 2008.

[47] A. Garcia-Cattaneo, F. X. Gobert, M. Muller et al., "Cleavage of Toll-like receptor 3 by cathepsins B and $\mathrm{H}$ is essential for signaling," Proceedings of the National Academy of Sciences, vol. 109, no. 23, pp. 9053-9058, 2012.

[48] M. E. Peter, A. V. Kubarenko, A. N. R. Weber, and A. H. Dalpke, "Identification of an $\mathrm{N}$-terminal recognition site in TLR9 that contributes to CpG-DNA-mediated receptor activation," The Journal of Immunology, vol. 182, no. 12, pp. 7690-7697, 2009.

[49] M. Onji, A. Kanno, S. Saitoh et al., "An essential role for the N-terminal fragment of Toll-like receptor 9 in DNA sensing," Nature Communications, vol. 4, no. 1, 2013.

[50] A. Bagchi, E. A. Herrup, H. S. Warren et al., "MyD88-dependent and MyD88-independent pathways in synergy, priming, and tolerance between TLR agonists," Journal of Immunology, vol. 178, no. 2, pp. 1164-1171, 2007.

[51] S. C. Lin, Y. C. Lo, and H. Wu, "Helical assembly in the MyD88-IRAK4-IRAK2 complex in TLR/IL-1R signalling," Nature, vol. 465, no. 7300, pp. 885-890, 2010.

[52] M. Bao and Y. J. Liu, "Regulation of TLR7/9 signaling in plasmacytoid dendritic cells," Protein \& Cell, vol. 4, no. 1, pp. 40-52, 2013.

[53] A. Dalpke, J. Frank, M. Peter, and K. Heeg, "Activation of toll-like receptor 9 by DNA from different bacterial species," Infection and Immunity, vol. 74, no. 2, pp. 940-946, 2006. 
[54] D. De Nardo, C. M. De Nardo, T. Nguyen, J. A. Hamilton, and G. M. Scholz, "Signaling crosstalk during sequential TLR4 and TLR9 activation amplifies the inflammatory response of mouse macrophages," Journal of Immunology, vol. 183, no. 12, pp. 8110-8118, 2009.

[55] E. Bourke, D. Bosisio, J. Golay, N. Polentarutti, and A. Mantovani, "The toll-like receptor repertoire of human B lymphocytes: inducible and selective expression of TLR9 and TLR10 in normal and transformed cells," Blood, vol. 102, no. 3, pp. 956-963, 2003.

[56] V. J. Sindhava, M. A. Oropallo, K. Moody et al., "A TLR9dependent checkpoint governs B cell responses to DNAcontaining antigens," The Journal of Clinical Investigation, vol. 127, no. 5, pp. 1651-1663, 2017.

[57] L. Sun, J. Wu, F. Du, X. Chen, and Z. J. Chen, "Cyclic GMPAMP synthase is a cytosolic DNA sensor that activates the type I interferon pathway," Science, vol. 339, no. 6121, pp. 786-791, 2013.

[58] D. L. Burdette, K. M. Monroe, K. Sotelo-Troha et al., "STING is a direct innate immune sensor of cyclic di-GMP," Nature, vol. 478, no. 7370, pp. 515-518, 2011.

[59] A. Ablasser and Z. J. Chen, "cGAS in action: expanding roles in immunity and inflammation," Science, vol. 363, no. 6431, 2019.

[60] H. Sui, M. Zhou, H. Imamichi et al., "STING is an essential mediator of the Ku70-mediated production of IFN- $\lambda 1$ in response to exogenous DNA," Science Signaling, vol. 10, no. $488,2017$.

[61] H. Ishikawa and G. N. Barber, "STING is an endoplasmic reticulum adaptor that facilitates innate immune signalling," Nature, vol. 455, pp. 674-678, 2008.

[62] T. Abe and G. N. Barber, "Cytosolic-DNA-Mediated, STING-Dependent Proinflammatory Gene Induction Necessitates Canonical NF- $\kappa$ B Activation through TBK1," Journal of Virology, vol. 88, no. 10, pp. 5328-5341, 2014.

[63] Y. Wu, Q. Wei, and J. Yu, "The cGAS/STING pathway: a sensor of senescence-associated DNA damage and trigger of inflammation in early age-related macular degeneration," Clinical Interventions in Aging, vol. 14, pp. 1277-1283, 2019.

[64] E. J. Diner, D. L. Burdette, S. C. Wilson et al., "The innate immune DNA sensor cGAS produces a noncanonical cyclic dinucleotide that activates human STING," Cell Reports, vol. 3, no. 5, pp. 1355-1361, 2013.

[65] N. Le Bert, A. R. Lam, S. S. Ho, Y. J. Shen, M. M. Liu, and S. Gasser, "STING-dependent cytosolic DNA sensor pathways regulate NKG2D ligand expression," OncoImmunology, vol. 3, no. 6, article e29259, 2014.

[66] Z. M. Parker, A. A. Murphy, and D. A. Leib, "Role of the DNA sensor STING in protection from lethal infection following corneal and intracerebral challenge with herpes simplex virus 1," Journal of Virology, vol. 89, no. 21, pp. 11080-11091, 2015

[67] Y. Hou, H. Liang, E. Rao et al., "Non-canonical NF- $\kappa$ B Antagonizes STING Sensor-Mediated DNA Sensing in Radiotherapy," Immunity, vol. 49, no. 3, pp. 490-503.e4, 2018.

[68] J. Ahn, D. Gutman, S. Saijo, and G. N. Barber, "STING manifests self DNA-dependent inflammatory disease," Proceedings of the National Academy of Sciences of the United States of America, vol. 109, no. 47, pp. 19386-19391, 2012.

[69] B. Larkin, V. Ilyukha, M. Sorokin, A. Buzdin, E. Vannier, and A. Poltorak, "Cutting edge: activation of STING in T cells induces type I IFN responses and cell death," Journal of Immunology, vol. 199, no. 2, pp. 397-402, 2017.

[70] C.-H. A. Tang, J. A. Zundell, S. Ranatunga et al., "Agonistmediated activation of STING induces apoptosis in malignant B cells," Cancer Research, vol. 76, no. 8, pp. 21372152, 2016.

[71] Y. Cheng, Y. Liu, Y. Wang et al., "Chicken DNA virus sensor DDX41 activates IFN- $\beta$ signaling pathway dependent on STING," Developmental and Comparative Immunology, vol. 76, pp. 334-342, 2017.

[72] H. Ishikawa, Z. Ma, and G. N. Barber, "STING regulates intracellular DNA-mediated, type I interferon-dependent innate immunity," Nature, vol. 461, no. 7265, pp. 788-792, 2009.

[73] J. Bai and F. Liu, "The cGAS-cGAMP-STING pathway: a molecular link between immunity and metabolism," Diabetes, vol. 68, no. 6, pp. 1099-1108, 2019.

[74] L. E. A. Ludlow, R. W. Johnstone, and C. J. P. Clarke, "The HIN-200 family: more than interferon-inducible genes?" Experimental Cell Research, vol. 308, no. 1, pp. 1-17, 2005.

[75] S. E. Warren, A. Armstrong, M. K. Hamilton et al., "Cutting edge: cytosolic bacterial DNA activates the inflammasome via Aim2," The Journal of Immunology, vol. 185, no. 2, pp. 818-821, 2010.

[76] T. Fernandes-Alnemri, J. W. Yu, P. Datta, J. Wu, and E. S. Alnemri, "AIM2 activates the inflammasome and cell death in response to cytoplasmic DNA," Nature, vol. 458, no. 7237, pp. 509-513, 2009.

[77] B. Hu, C. Jin, H. B. Li et al., “The DNA-sensing AIM2 inflammasome controls radiation-induced cell death and tissue injury," Science, vol. 354, no. 6313, pp. 765-768, 2016.

[78] D. A. Muruve, V. Petrilli, A. K. Zaiss et al., "The inflammasome recognizes cytosolic microbial and host DNA and triggers an innate immune response," Nature, vol. 452, no. 7183, pp. 103-107, 2008.

[79] T. L. Roberts, A. Idris, J. A. Dunn et al., "HIN-200 proteins regulate caspase activation in response to foreign cytoplasmic DNA," Science, vol. 323, no. 5917, pp. 10571060,2009

[80] V. Hornung, A. Ablasser, M. Charrel-Dennis et al., "AIM2 recognizes cytosolic dsDNA and forms a caspase-1activating inflammasome with ASC," Nature, vol. 458, no. 7237, pp. 514-518, 2009.

[81] T. Burckstummer, C. Baumann, S. Bluml et al., "An orthogonal proteomic-genomic screen identifies AIM2 as a cytoplasmic DNA sensor for the inflammasome," Nature Immunology, vol. 10, no. 3, pp. 266-272, 2009.

[82] J. M. Kahlenberg and M. J. Kaplan, "Little peptide, big effects: the role of LL-37 in inflammation and autoimmune disease," The Journal of Immunology, vol. 191, no. 10, pp. 4895-4901, 2013.

[83] L. Unterholzner, S. E. Keating, M. Baran et al., "IFI16 is an innate immune sensor for intracellular DNA," Nature Immunology, vol. 11, no. 11, pp. 997-1004, 2010.

[84] A. Roy, A. Ghosh, B. Kumar, and B. Chandran, "IFI16, a nuclear innate immune DNA sensor, mediates epigenetic silencing of herpesvirus genomes by its association with H3K9 methyltransferases SUV39H1 and GLP," Elife, vol. 8, 2019.

[85] S. A. Stratmann, S. R. Morrone, A. M. van Oijen, and J. Sohn, "The innate immune sensor IFI16 recognizes foreign DNA in 
the nucleus by scanning along the duplex," eLife, vol. 4, article e11721, 2015.

[86] S. Veeranki and D. Choubey, "Interferon-inducible p200family protein IFI16, an innate immune sensor for cytosolic and nuclear double-stranded DNA: regulation of subcellular localization," Molecular Immunology, vol. 49, no. 4, pp. 567571, 2012.

[87] J. F. Almine, C. A. O'Hare, G. Dunphy et al., "IFI16 and cGAS cooperate in the activation of STING during DNA sensing in human keratinocytes," Nature Communications, vol. 8, no. 1, article 14392, 2017.

[88] G. Dunphy, S. M. Flannery, J. F. Almine et al., "Noncanonical Activation of the DNA Sensing Adaptor STING by ATM and IFI16 Mediates NF- $\kappa$ B Signaling after Nuclear DNA Damage," Molecular Cell, vol. 71, no. 5, pp. 745-760.e5, 2018.

[89] A. Takaoka, Z. Wang, M. K. Choi et al., "DAI (DLM-1/ZBP1) is a cytosolic DNA sensor and an activator of innate immune response," Nature, vol. 448, no. 7152, pp. 501-505, 2007.

[90] P. Yang, H. An, X. Liu et al., "The cytosolic nucleic acid sensor LRRFIP1 mediates the production of type I interferon via a $\beta$-catenin-dependent pathway," Nature Immunology, vol. 11, no. 6, pp. 487-494, 2010.

[91] A. Ablasser, F. Bauernfeind, G. Hartmann, E. Latz, K. A. Fitzgerald, and V. Hornung, "RIG-I-dependent sensing of poly(dA:dT) through the induction of an RNA polymerase III-transcribed RNA intermediate," Nature Immunology, vol. 10, no. 10, pp. 1065-1072, 2009.

[92] Y. H. Chiu, J. B. Macmillan, and Z. J. Chen, "RNA polymerase III detects cytosolic DNA and induces type I interferons through the RIG-I pathway," Cell, vol. 138, no. 3, pp. 576591, 2009.

[93] T. Kim, S. Pazhoor, M. Bao et al., "Aspartate-glutamate-alanine-histidine box motif (DEAH)/RNA helicase A helicases sense microbial DNA in human plasmacytoid dendritic cells," Proceedings of the National Academy of Sciences of the United States of America, vol. 107, no. 34, pp. 1518115186, 2010.

[94] X. Zhang, T. W. Brann, M. Zhou et al., "Cutting edge: Ku70 is a novel cytosolic DNA sensor that induces type III rather than type I IFN," Journal of Immunology, vol. 186, no. 8, pp. 4541-4545, 2011.

[95] S. Murakami, N. Morimoto, T. Kono, M. Sakai, and J. I. Hikima, "Molecular characterization and expression of the teleost cytosolic DNA sensor genes cGAS, LSm14A, DHX9, and DHX36 in Japanese medaka, Oryzias latipes," Developmental \& Comparative Immunology, vol. 99, article 103402, 2019.

[96] M. Yoneyama, M. Kikuchi, K. Matsumoto et al., "Shared and unique functions of the $\mathrm{DExD} / \mathrm{H}$-box helicases RIG-I, MDA5, and LGP2 in antiviral innate immunity," Journal of Immunology, vol. 175, no. 5, pp. 2851-2858, 2005.

[97] L. Wang, M. Wen, and X. Cao, "Nuclear hnRNPA2B1 initiates and amplifies the innate immune response to DNA viruses," Science, vol. 365, no. 6454, 2019.

[98] K. C. Barnett, J. M. Coronas-Serna, W. Zhou et al., "Phosphoinositide interactions position cGAS at the plasma membrane to ensure efficient distinction between self- and viral DNA,” Cell, vol. 176, no. 6, pp. 1432-1446.e11, 2019.

[99] H. Jiang, X. Xue, S. Panda et al., "Chromatin-bound cGAS is an inhibitor of DNA repair and hence accelerates genome destabilization and cell death," The EMBO Journal, vol. 38, no. 21, article e102718, 2019.

[100] T. L. Roberts, M. L. Turner, J. A. Dunn et al., "B cells do not take up bacterial DNA: an essential role for antigen in exposure of DNA to toll-like receptor-9," Immunology and Cell Biology, vol. 89, no. 4, pp. 517-525, 2011.

[101] H. Yang, H. Liu, Q. Zeng et al., "Inhibition of HMGB1/ RAGE-mediated endocytosis by HMGB1 antagonist box A, anti-HMGB1 antibodies, and cholinergic agonists suppresses inflammation," Molecular Medicine, vol. 25, no. 1, 2019.

[102] H. E. Hofland, L. Shephard, and S. M. Sullivan, "Formation of stable cationic lipid/DNA complexes for gene transfer," Proceedings of the National Academy of Sciences, vol. 93, no. 14, pp. 7305-7309, 1996.

[103] N. J. Bitto, R. Chapman, S. Pidot et al., "Bacterial membrane vesicles transport their DNA cargo into host cells," Scientific Reports, vol. 7, no. 1, 2017.

[104] T. Li and Z. J. Chen, "The cGAS-cGAMP-STING pathway connects DNA damage to inflammation, senescence, and cancer," The Journal of Experimental Medicine, vol. 215, no. 5, pp. 1287-1299, 2018.

[105] A. Li, M. Yi, S. Qin, Y. Song, Q. Chu, and K. Wu, "The cGAScGAMP-STING pathway connects DNA damage to inflammation, senescence, and cancer," Journal of Hematology \& Oncology, vol. 12, no. 1, 2019.

[106] D. Li, R. Wu, W. Guo et al., "STING-mediated IFI16 degradation negatively controls type I interferon production," Cell Reports, vol. 29, no. 5, pp. 1249-1260.e4, 2019.

[107] H. Chen, H. Sun, F. You et al., "Activation of STAT6 by STING is critical for antiviral innate immunity," Cell, vol. 147, no. 2, pp. 436-446, 2011.

[108] D. Liu, H. Wu, C. Wang et al., "STING directly activates autophagy to tune the innate immune response," Cell Death and Differentiation, vol. 26, no. 9, pp. 1735-1749, 2019.

[109] M. Motwani, S. Pesiridis, and K. A. Fitzgerald, "DNA sensing by the cGAS-STING pathway in health and disease," Nature Reviews. Genetics, vol. 20, no. 11, pp. 657-674, 2019.

[110] S. Liu and W. Guan, "STING signaling promotes apoptosis, necrosis, and cell death: an overview and update," Mediators of Inflammation, vol. 2018, Article ID 1202797, 4 pages, 2018.

[111] A. Sze, S. M. Belgnaoui, D. Olagnier, R. Lin, J. Hiscott, and J. van Grevenynghe, "Host restriction factor SAMHD1 limits human T cell leukemia virus type 1 infection of monocytes via STING-mediated apoptosis," Cell Host \& Microbe, vol. 14, no. 4, pp. 422-434, 2013.

[112] J. T. Qiao, C. Cui, L. Qing et al., "Activation of the STINGIRF3 pathway promotes hepatocyte inflammation, apoptosis and induces metabolic disorders in nonalcoholic fatty liver disease," Metabolism, vol. 81, pp. 13-24, 2018.

[113] J. Petrasek, A. Iracheta-Vellve, T. Csak et al., "STING-IRF3 pathway links endoplasmic reticulum stress with hepatocyte apoptosis in early alcoholic liver disease," Proceedings of the National Academy of Sciences, vol. 110, no. 41, pp. 1654416549, 2013.

[114] N. Li, H. Zhou, H. Wu et al., "STING-IRF3 contributes to lipopolysaccharide-induced cardiac dysfunction, inflammation, apoptosis and pyroptosis by activating NLRP3," Redox Biology, vol. 24, article 101215, 2019.

[115] M. M. Gaidt, T. S. Ebert, D. Chauhan et al., "The DNA inflammasome in human myeloid cells is initiated by a 
STING-cell death program upstream of NLRP3," Cell, vol. 171, no. 5, pp. 1110-1124.e18, 2017.

[116] K. McArthur, L. W. Whitehead, J. M. Heddleston et al., "BAK/BAX macropores facilitate mitochondrial herniation and mtDNA efflux during apoptosis," Science, vol. 359, no. $6378,2018$.

[117] S. Cornelie, E. Wiel, N. Lund et al., "Cytosine-phosphate-guanine (CpG) motifs are sensitizing agents for lipopolysaccharide in toxic shock model," Intensive Care Medicine, vol. 28, no. 9, pp. 1340-1347, 2002.

[118] G. Hartmann and A. M. Krieg, "CpG DNA and LPS induce distinct patterns of activation in human monocytes," Gene Therapy, vol. 6, no. 5, pp. 893-903, 1999.

[119] J. J. Gao, V. Diesl, T. Wittmann et al., "Bacterial LPS and CpG DNA differentially induce gene expression profiles in mouse macrophages," Journal of Endotoxin Research, vol. 9, no. 4, pp. 237-243, 2003.

[120] A. K. Yi, J. G. Yoon, S. C. Hong, T. W. Redford, and A. M. Krieg, "Lipopolysaccharide and CpG DNA synergize for tumor necrosis factor- $\alpha$ production through activation of $\mathrm{NF}-\kappa \mathrm{B}$," International Immunology, vol. 13 , no. 11 , pp. 1391-1404, 2001.

[121] J. J. Gao, E. G. Zuvanich, Q. Xue, D. L. Horn, R. Silverstein, and D. C. Morrison, "Cutting Edge: Bacterial DNA and LPS Act in Synergy in Inducing Nitric Oxide Production in RAW 264.7 Macrophages," Journal of Immunology, vol. 163, no. 8, pp. 4095-4099, 1999.

[122] S. L. McCoy, S. E. Kurtz, F. A. Hausman, D. R. Trune, R. M. Bennett, and S. H. Hefeneider, "Activation of RAW264.7 macrophages by bacterial DNA and lipopolysaccharide increases cell surface DNA binding and internalization," The Journal of Biological Chemistry, vol. 279, no. 17, pp. 17217-17223, 2004.

[123] J. J. Gao, Q. Xue, C. J. Papasian, and D. C. Morrison, "Bacterial DNA and Lipopolysaccharide Induce Synergistic Production of TNF- $\alpha$ Through a Post-Transcriptional Mechanism," Journal of Immunology, vol. 166, no. 11, pp. 6855-6860, 2001.

[124] R. Silverstein, "D-galactosamine lethality model: scope and limitations," Journal of Endotoxin Research, vol. 10, no. 3, pp. 147-162, 2004.

[125] J. M. Alcorn, J. Fierer, and M. Chojkier, "The acute-phase response protects mice from D-galactosamine sensitization to endotoxin and tumor necrosis factor- $\alpha$," Hepatology, vol. 15, no. 1, pp. 122-129, 1992.

[126] A. K. Yi, H. Yoon, J. E. Park, B. S. Kim, H. J. Kim, and A. Martinez-Hernandez, "CpG DNA-mediated induction of acute liver injury in D-galactosamine-sensitized mice: the mitochondrial apoptotic pathway-dependent death of hepatocytes," The Journal of Biological Chemistry, vol. 281, no. 21, pp. 15001-15012, 2006.

[127] R. Seki, “Toll-Like Receptor Ligand-Induced Liver Injury in D-Galactosamine-Sensitized Mice: Differences between TLR7/8 and TLR9 Ligands, Cytokine Patterns, and CrossTolerance Induction by TLR2 Ligand Pretreatment," Journal of Immunology Research, vol. 2017, Article ID 9653793, 10 pages, 2017.

[128] S. Zhang, N. Yang, S. Ni et al., "Pretreatment of lipopolysaccharide (LPS) ameliorates D-GalN/LPS induced acute liver failure through TLR4 signaling pathway," International Journal of Clinical and Experimental Pathology, vol. 7, no. 10, pp. 6626-6634, 2014.
[129] M. A. Freudenberg, D. Keppler, and C. Galanos, "Requirement for lipopolysaccharide-responsive macrophages in galactosamine-induced sensitization to endotoxin," Infection and Immunity, vol. 51, no. 3, pp. 891-895, 1986.

[130] C. Galanos, M. A. Freudenberg, and W. Reutter, "Galactosamine-induced sensitization to the lethal effects of endotoxin," Proceedings of the National Academy of Sciences of the United States of America, vol. 76, no. 11, pp. 5939-5943, 1979.

[131] T. Fernandes-Alnemri, J. W. Yu, C. Juliana et al., "The AIM2 inflammasome is critical for innate immunity to Francisella tularensis," Nature Immunology, vol. 11, no. 5, pp. 385-393, 2010.

[132] T. Sparwasser, T. Miethke, G. Lipford et al., "Bacterial DNA causes septic shock," Nature, vol. 386, no. 6623, pp. 336337, 1997.

[133] G. Plitas, B. M. Burt, H. M. Nguyen, Z. M. Bamboat, and R. P. DeMatteo, "Toll-like receptor 9 inhibition reduces mortality in polymicrobial sepsis," The Journal of Experimental Medicine, vol. 205, no. 6, pp. 1277-1283, 2008.

[134] X. Liu, X. Zheng, N. Wang et al., "Kukoamine B, a novel dual inhibitor of LPS and CPG DNA, is a potential candidate for sepsis treatment," British Journal of Pharmacology, vol. 162, no. 6, pp. 1274-1290, 2011.

[135] S. Tasaka, H. Kamata, K. Miyamoto et al., "Intratracheal synthetic CpG oligodeoxynucleotide causes acute lung injury with systemic inflammatory response," Respiratory Research, vol. 10, no. 1, 2009.

[136] P. Knuefermann, G. Baumgarten, A. Koch et al., "CpG oligonucleotide activates Toll-like receptor 9 and causes lung inflammation in vivo," Respiratory Research, vol. 8, no. 1, 2007.

[137] H. Yamada, K. J. Ishii, and D. M. Klinman, "Suppressive oligodeoxynucleotides inhibit CpG-induced inflammation of the mouse lung," Critical Care Medicine, vol. 32, no. 10, pp. 2045-2049, 2004.

[138] K. Itagaki, Y. Adibnia, S. Sun et al., "Bacterial DNA induces pulmonary damage via TLR-9 through cross-talk with neutrophils," Shock, vol. 36, no. 6, pp. 548-552, 2011.

[139] D. Klinman, H. Shirota, D. Tross, T. Sato, and S. Klaschik, "Synthetic oligonucleotides as modulators of inflammation," Journal of Leukocyte Biology, vol. 84, no. 4, pp. 958-964, 2008.

[140] D. M. Klinman, "Immunotherapeutic uses of CpG oligodeoxynucleotides," Nature Reviews. Immunology, vol. 4, no. 4, pp. 249-259, 2004.

[141] C. Bode, G. Zhao, F. Steinhagen, T. Kinjo, and D. M. Klinman, "CpG DNA as a vaccine adjuvant," Expert Review of Vaccines, vol. 10, pp. 499-511, 2014.

[142] M. D. Luyer, W. A. Buurman, M. Hadfoune et al., "Exposure to bacterial DNA before hemorrhagic shock strongly aggravates systemic inflammation and gut barrier loss via an IFN-gamma-dependent route," Annals of Surgery, vol. 245, no. 5, pp. 795-802, 2007.

[143] J. C. Deng, T. A. Moore, M. W. Newstead, X. Zeng, A. M. Krieg, and T. J. Standiford, "CpG oligodeoxynucleotides stimulate protective innate immunity against pulmonary Klebsiella infection," Journal of Immunology, vol. 173, no. 8, pp. 5148-5155, 2004.

[144] S. Ribes, T. Meister, M. Ott et al., "Intraperitoneal prophylaxis with $\mathrm{CpG}$ oligodeoxynucleotides protects neutropenic 
mice against intracerebral Escherichia coli K1 infection," Journal of Neuroinflammation, vol. 11, no. 1, 2014.

[145] T. H. Kim, J. Park, D. Kim et al., "Anti-Bacterial Effect of CpG-DNA Involves Enhancement of the Complement Systems," International Journal of Molecular Sciences, vol. 20, no. 14, 2019.

[146] T. H. Kim, D. Kim, A. Gautam et al., "CpG-DNA exerts antibacterial effects by protecting immune cells and producing bacteria-reactive antibodies," Scientific Reports, vol. 8, no. 1, article 16236, 2018.

[147] T. H. Kim, D. Kim, H. Lee et al., "CpG-DNA induces bacteria-reactive IgM enhancing phagocytic activity against Staphylococcus aureus infection," BMB Reports, vol. 52, no. 11, pp. 635-640, 2019.

[148] H. M. Wu, J. Wang, B. Zhang, L. Fang, K. Xu, and R. Y. Liu, "CpG-ODN promotes phagocytosis and autophagy through JNK/P38 signal pathway in Staphylococcus aureus-stimulated macrophage," Life Sciences, vol. 161, pp. 51-59, 2016.

[149] J. V. Villarreal, C. Jungfer, U. Obst, and T. Schwartz, "DNase I and Proteinase K eliminate DNA from injured or dead bacteria but not from living bacteria in microbial reference systems and natural drinking water biofilms for subsequent molecular biology analyses," Journal of Microbiological Methods, vol. 94, no. 3, pp. 161-169, 2013.

[150] V. V. Tetz and G. V. Tetz, "Effect of extracellular DNA destruction by DNase I on characteristics of forming biofilms," DNA and Cell Biology, vol. 29, no. 8, pp. 399-405, 2010.

[151] G. B. Lim, "DNases prevent clots formed by neutrophil extracellular traps," Nature Reviews. Cardiology, vol. 15, no. 2, 2018.

[152] P. A. Keyel, "Dnases in health and disease," Developmental Biology, vol. 429, no. 1, pp. 1-11, 2017.

[153] X. Huang and Y. Yang, "Targeting the TLR9-MyD88 pathway in the regulation of adaptive immune responses," Expert Opinion on Therapeutic Targets, vol. 14, no. 8, pp. 787-796, 2010.

[154] A. M. Krieg, "Development of TLR9 agonists for cancer therapy," The Journal of Clinical Investigation, vol. 117, no. 5, pp. 1184-1194, 2007.

[155] M. A. Anwar, M. Shah, J. Kim, and S. Choi, "Recent clinical trends in Toll-like receptor targeting therapeutics," Medicinal Research Reviews, vol. 39, no. 3, pp. 1053-1090, 2019.

[156] J. K. Dowling and A. Mansell, "Toll-like receptors: the swiss army knife of immunity and vaccine development," Clinical \& Translational Immunology, vol. 5, no. 5, 2016.

[157] D. van Duin, R. Medzhitov, and A. C. Shaw, "Triggering TLR signaling in vaccination," Trends in Immunology, vol. 27, no. 1, pp. 49-55, 2006.

[158] Q. Zhao, Y. Wei, S. J. Pandol, L. Li, and A. Habtezion, "STING signaling promotes inflammation in experimental acute pancreatitis," Gastroenterology, vol. 154, no. 6, pp. 1822-1835.e2, 2018.

[159] D. Chandra, W. Quispe-Tintaya, A. Jahangir et al., "STING ligand c-di-GMP improves cancer vaccination against metastatic breast cancer," Cancer Immunology Research, vol. 2, no. 9, pp. 901-910, 2014.

[160] C. Sheridan, "Drug developers switch gears to inhibit STING," Nature Biotechnology, vol. 37, no. 3, pp. 199-201, 2019.

[161] W. Jing, D. McAllister, E. P. Vonderhaar et al., "STING agonist inflames the pancreatic cancer immune microenvi- ronment and reduces tumor burden in mouse models," Journal for Immunotherapy of Cancer, vol. 7, no. 1, 2019.

[162] X. Feng, D. Liu, Z. Li, and J. Bian, "Bioactive modulators targeting STING adaptor in cGAS-STING pathway," Drug Discovery Today, vol. 25, no. 1, pp. 230-237, 2020.

[163] C. Uggenti and Y. J. Crow, "Taking the STING out of inflammation," Nature Reviews Rheumatology, vol. 14, no. 9, pp. 508-509, 2018.

[164] S. Iurescia, D. Fioretti, and M. Rinaldi, "Targeting cytosolic nucleic acid-sensing pathways for cancer immunotherapies," Frontiers in Immunology, vol. 9, p. 711, 2018.

[165] K. Kawa, H. Tsutsui, R. Uchiyama et al., "IFN- $\gamma$ is a master regulator of endotoxin shock syndrome in mice primed with heat-killed Propionibacterium acnes," International Immunology, vol. 22, no. 3, pp. 157-166, 2010.

[166] N. Lacerda-Queiroz, N. Riteau, R. T. Eastman et al., "Mechanism of splenic cell death and host mortality in a Plasmodium yoelii malaria model," Scientific Reports, vol. 7, no. 1, article 10438, 2017.

[167] B. D. Car, V. M. Eng, B. Schnyder et al., "Interferon gamma receptor deficient mice are resistant to endotoxic shock," The Journal of Experimental Medicine, vol. 179, no. 5, pp. 1437-1444, 1994.

[168] F. P. Heinzel, "The role of IFN-gamma in the pathology of experimental endotoxemia," Journal of Immunology, vol. 145, no. 9, pp. 2920-2924, 1990.

[169] M. M. Zaman, K. Masuda, K. K. Nyati et al., "Arid5a exacerbates IFN- $\gamma$-mediated septic shock by stabilizing T-bet mRNA," Proceedings of the National Academy of Sciences of the United States of America, vol. 113, no. 41, pp. 1154311548, 2016. 